\title{
Single-hole transistor in a $p$-Si/SiGe quantum well
}

\author{
U. Dötsch, U. Gennser, C. David, G. Dehlinger, and D. Grützmacher \\ Paul Scherrer Institute, 5232 Villigen PSI, Switzerland
}

T. Heinzel, ${ }^{\text {a) }}$ S. Lüscher, and K. Ensslin

Solid State Physics Laboratory, ETH Zürich, 8093 Zürich, Switzerland

(Received 15 September 2000; accepted for publication 16 November 2000)

\begin{abstract}
A single-hole transistor is patterned in a $p$-Si/SiGe quantum well by applying voltages to nanostructured top gate electrodes. Gating is achieved by oxidizing the etched semiconductor surface and the mesa walls before evaporation of the top gates. Pronounced Coulomb blockade effects are observed at small coupling of the transistor island to source and drain. (C) 2001 American Institute of Physics. [DOI: 10.1063/1.1342040]
\end{abstract}

Transport through small metallic islands, defined in semiconductor heterostructures, has been of considerable interest recently. ${ }^{1}$ When the island is only weakly coupled to reservoirs via tunnel barriers, the Coulomb blockade (CB) effect determines the carrier transport. In such islands, the interplay between interactions and size quantization can be studied. A large number of experiments have investigated the properties of single electron transistors made out of conventional metals, and of high mobility, low density twodimensional electron gases, defined in mostly $\mathrm{Ga}[\mathrm{Al}] \mathrm{As}$ or $\mathrm{Si}$, among other materials. ${ }^{1}$ Single hole transistors out of $p$-Si/SiGe quantum wells are of particular interest for several reasons: (i) the material has a large $g$ factor of $g_{\text {eff }}=6.7$ and a large effective mass $\left(m^{*}=0.25 m_{e}\right)$, (ii) the interaction parameter $r_{S}$, defined as the ratio between the Coulomb energy of two holes at their average separation, and the Fermi energy, is very large, i.e., $r_{S} \approx 4$ for typical carrier densities, and (iii) the properties of the quantum well are strongly influenced by biaxial strain, due to the lattice mismatch at the $\mathrm{Si} / \mathrm{SiGe}$ interface.

However, $p$-SiGe has been notoriously difficult to gate, due to leakage current currents across the Schottky barrier. ${ }^{2,3}$ Recently, progress has been reported in this respect. Emeleus et $a l .{ }^{4}$ have tuned the hole density in a $p-\mathrm{Si} / \mathrm{SiGe}$ quantum well using a homogeneous backgate. Homogeneous top gating of this material has been reported as well. ${ }^{5}$ In those samples, the two-dimensional hole gas (2DHG) is buried very deeply below the surface, which limits the pattern transfer into the 2DHG. The fabrication of any kind of gated nanostructure in $p$-Si/SiGe has not been reported yet. It should be noted that small islands and wires have been patterned out of $p-\mathrm{Si}_{\mathrm{Si}} \mathrm{Si}_{1-x} \mathrm{Ge}_{x} / \mathrm{Si}$ resonant tunneling structures by etching. ${ }^{6}$ Furthermore, single hole charging effects have been observed in $p-\mathrm{Si}^{7}$ and in two-terminal, $\delta$-doped SiGe devices. ${ }^{8}$

Here, we present the fabrication and characterization of a gated single hole transistor in a $p$-Si/SiGe quantum well. The gate leakage is reduced by oxidizing the etched surface prior to patterning of the top gates. At low temperatures, we observe clear single hole tunneling and CB oscillations.

${ }^{a)}$ Electronic mail: heinzel@solid.phys.ethz.ch
The samples have been grown by molecular beam epitaxy, and contain a $200 \AA \mathrm{Si}_{0.85} \mathrm{Ge}_{0.15}$ layer on top of an undoped Si substrate, a $150 \AA$ B -doped Si layer with a setback of $180 \AA$ from the well, and a $200 \AA$ undoped Si cap. The $\mathrm{Si}_{0.85} \mathrm{Ge}_{0.15}$ layer forms a triangular potential well for the two-dimensional hole gas [Fig. 1(a)]. Due to the lattice mismatch between $\mathrm{Si}$ and $\mathrm{Si}_{0.85} \mathrm{Ge}_{0.15}$, the heavy hole $\left(m_{J}\right.$ $= \pm 3 / 2)$ potential is split from the light hole $\left(m_{J}= \pm 1 / 2\right)$ potential, and ensures that the lowest occupied bound state has heavy hole character. The hole density of the ungated 2DHG is $p_{2 \mathrm{D}}=3.6 \times 10^{15} \mathrm{~m}^{-2}$, and an elastic mean free path of $l_{e}=100 \mathrm{~nm}$ is obtained from magnetoresistivity measurements.

Tests have indicated that in our samples, large leakage currents between the top gate and the 2DHG can flow across the mesa edge. Therefore, we have deposited a layer of $\mathrm{SiO}_{2}$ by thermal evaporation right after the reactive ion etch that defines the mesa, i.e., with the photoresist still on the mesa, Fig. 1(b). We have used this technique in earlier work for the fabrication of homogeneous gates. ${ }^{9}$ After lift-off of the oxide, the top gate $(10 \mathrm{~nm} \mathrm{Ti}$ and $20 \mathrm{~nm} \mathrm{Al})$ was patterned by electron beam lithography and thermal evaporation. This sequence of technology steps reduces leakage currents in our samples significantly.

The geometry of the gates is crucial. For grounded Ti/Al gates in the submicron regime, the 2DHG is depleted under-

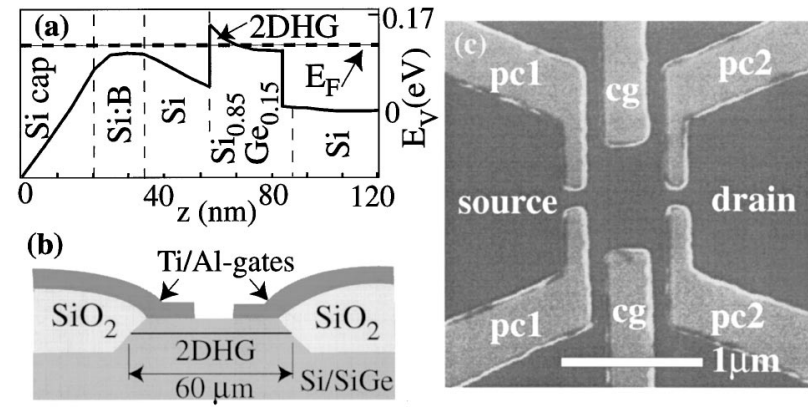

FIG. 1. (a) Top of the valence band of the Si/SiGe quantum well. The hole gas resides at the $\mathrm{Si}_{2} \mathrm{Si}_{0.85} \mathrm{Ge}_{0.15}$ interface $53 \mathrm{~nm}$ below the surface. (b) Schematic cross section through the processed heterostructure. In order to reduce leakage currents, the mesa edges are separated from the top gate by a $\mathrm{SiO}_{2}$ layer. (c) Scanning electron micrograph of the gate geometry. The $\mathrm{Ti} / \mathrm{Al}$ gates appear as bright areas. 

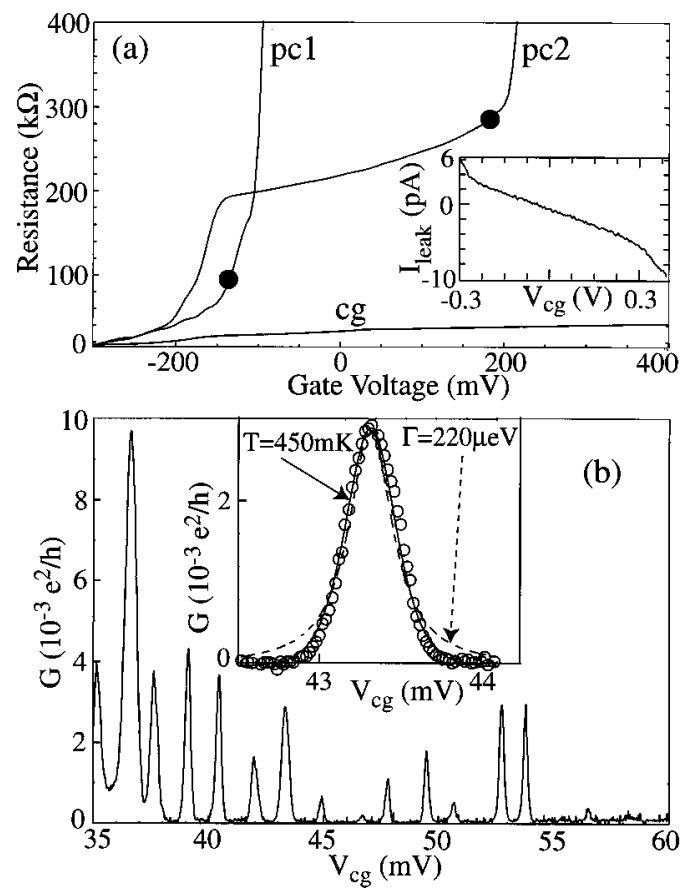

FIG. 2. (a) Resistance of the three split gates as a function of the gate voltages $V_{\mathrm{pc} 1}, V_{\mathrm{pc} 2}$, and $V_{\mathrm{cg}}$, respectively. The circles denote the working points of gates pc1 and pc2 for all subsequent measurements. Inset: leakage current of gate cg as a function of $V_{\mathrm{cg}}$, which is typical for all gates. (b) Conductance $G$ through the island as a function of $V_{\mathrm{cg}}$, showing CB oscillations, taken under $V_{\text {sd }}=100 \mu \mathrm{V}$. Inset: blow-up of the peak at $V_{\text {cg }}$ $=43 \mathrm{mV}$ (open circles), fitted to a Lorentzian (dashed line), and to a thermally broadened CB resonance (solid line). For all measurements, the temperature was $90 \mathrm{mK}$.

neath, with a lateral depletion length of roughly $100 \mathrm{~nm}$. This depletion is probably caused by strain and pinning on defects, which complicates a simulation of the device. Preliminary experimental studies on split gates with various openings $w$ between the two electrodes were used to develop the gate geometry. For $w<100 \mathrm{~nm}$, we were unable to open the channel between the split gates without simultaneously populating the regions below the gates. For $w>250 \mathrm{~nm}$, the channels could not be pinched off. Also, the pinch-off voltage of split gates with identical geometry can be quite different.

In Fig. 1(c), the island geometry is shown. Three split gate electrodes (point contact gates pc1, pc2, as well as the center gates $\mathrm{cg}$ ) define the dot with a lithographic size of $500 \mathrm{~nm} \times 800 \mathrm{~nm}$. For $\mathrm{pc} 1$ and $\mathrm{pc} 2, w=150 \mathrm{~nm}$ was chosen.

The measurements have been carried out in a ${ }^{3} \mathrm{He} /{ }^{4} \mathrm{He}$ dilution refrigerator with a base temperature of $90 \mathrm{mK}$. dc voltages $V_{\text {sd }}$ are applied between source and drain, and the current is measured with a resolution of $500 \mathrm{fA}$.

We have studied two different quantum dots of identical geometry and performed several cooldowns, all leading to similar results. Here, we focus on measurements from one device, taken in a single cooldown.

Split gates pc1 and pc2 are used to adjust the coupling of the island to source and drain [Fig. 2(a)]. Due to the small elastic mean free path, conductance quantization is not observed. The leakage currents measured at the center gates is shown in the inset of Fig. 2(a). For temperatures between 0.1 and $2 \mathrm{~K}$ investigated here, neither the resistance of the split
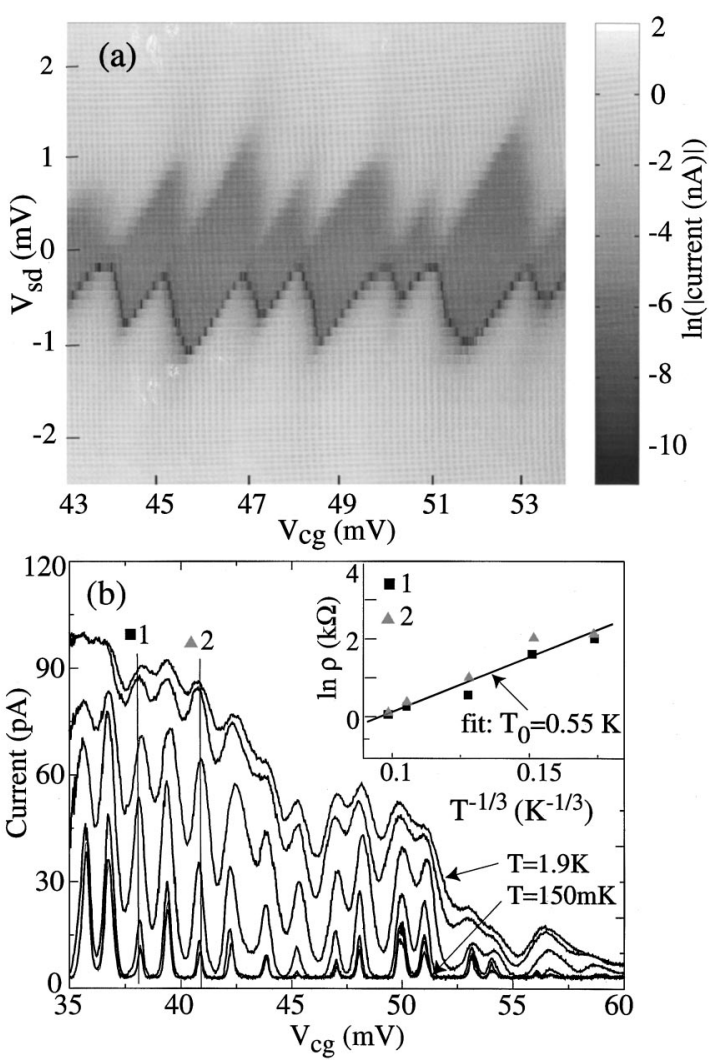

FIG. 3. (a) The Coulomb diamonds in a logarithmic gray scale plot. (b) Temperature dependence of the CB resonances. Besides a thermal smearing, a striking overall increase of $G$ is observed as $T$ increases. The inset shows $\ln (\rho)$ as a function of $T^{-1 / 3}$ at gate voltages 1 and 2, including a fit of the full squares. The approximately linear behavior indicates thermally activated transport through the island.

gates, nor the leakage currents revealed any temperature dependence within experimental accuracy.

At sufficiently large split gate voltages $V_{\mathrm{pc} 1}$ and $V_{\mathrm{pc} 2}$ (the working points are chosen to be $V_{\mathrm{pc} 1}=-140 \mathrm{mV}$ and $V_{\mathrm{pc} 2}$ $=180 \mathrm{mV}$; the corresponding leakage currents are 0.5 and $-1 \mathrm{pA}$, respectively), CB oscillations are observed as a function of the center gate voltage $V_{\text {cg }}$ [Fig. 2(b)]. Here, $V_{\text {sd }}$ was $100 \mu \mathrm{V}$. The measured $\mathrm{CB}$ resonances can be fitted well to the expression describing a thermally smeared $\mathrm{CB}$ peak in the multilevel transport regime, i.e., $G\left(V_{\mathrm{cg}}, T_{h}\right)$ $=G_{\max } \cosh ^{-2}\left[\eta\left(V_{\mathrm{cg}}-V_{\max }\right) / 2.5 k_{B} T_{h}\right] .{ }^{1}$ Here, $G_{\max }$ and $V_{\max }$ denote the amplitude and the position of the CB resonance, respectively. The lever arm $\eta=d E / d\left(\delta V_{\mathrm{cg}}\right)$ ( $\delta V_{\mathrm{cg}}$ is the Coulomb blockade oscillation period and $E=\mathrm{eV}_{\text {sd }}$ is the energy inside the island) translates $V_{\mathrm{cg}}$ into energy and is obtained from measurements of the CB diamonds [Fig. 3(a)]. We find $\eta=0.68 \mathrm{eV} / \mathrm{V}$. As fit parameter, we obtain hole temperatures of $T_{h}=390-570 \mathrm{mK}$, depending on the peak. A Lorentzian, on the other hand, which is appropriate for homogeneously broadened resonances, fits significantly worse [inset in Fig. 2(b)]. The full widths at half maximum (FWHMs) thus fluctuate strongly from peak to peak, and furthermore saturate for bath temperatures below $400 \mathrm{mK}$, as well as for source-drain bias voltages below $V_{\mathrm{sd}}=120 \mu \mathrm{V}$. We conclude that neither the electron temperature, nor $V_{\text {sd }}$ determine the FWHM in this regime $\left(T<400 \mathrm{mK}\right.$ and $V_{\text {sd }}$ $<120 \mu \mathrm{V}$ ). Furthermore, we observe a pronounced pair modulation of the peak separations in this regime. Qualita- 
tively similar modulations are observed in both devices and in all cooldowns, although the detailed structure varies strongly. A measurement of the Coulomb diamonds, i.e., the current as a function of both $V_{\text {sd }}$ and $V_{\text {cg }}$ is shown in Fig. 3(a). The stability of the diamonds shows that there is little telegraph noise and switching at frequencies below $1 \mathrm{~Hz}$, the bandwidth of our setup. However, noise of higher frequencies may be substantial; gate voltage dependent charge rearrangements in the substrate may be present and could determine the FWHM of the CB peaks. Note also that the leakage currents are not negligible as compared to the current through the dot and may modify the line shape as well.

In Fig. 3(b), we show the temperature dependence of the $\mathrm{CB}$ oscillations. As the temperature increases, not only the $\mathrm{CB}$ oscillations are smeared out, but also a striking overall increase in $G$ is observed, which indicates thermally enhanced transport through the island. Since neither the conductance of the individual tunnel barriers, measured with positive voltages applied to the remaining gates, nor the leakage currents depend on temperature between $90 \mathrm{mK}$ and $2 \mathrm{~K}$, the observed overall increase of $G$ is entirely due to the island. Several scenarios could lead to such a behavior, such as variable-range hopping ${ }^{10}$ or Efros-Shklovskii hopping. ${ }^{11}$ Reasonable agreement with variable-range hopping (i.e., $\rho$ $\left.\propto \exp \left[\left(T_{0} / T\right)^{1 / 3}\right]\right)$ is found, and we can estimate the characteristic temperature from fits to $T_{0} \approx 0.55 \mathrm{~K}$. This result depends only weakly on $V_{\text {cg }}$. We cannot, however, distinguish between variable-range hopping and other possible hopping mechanisms, due to the limited range of temperatures.

Similar modulations of Coulomb diamonds have been observed in double dot systems for weak coupling between the dots. ${ }^{12}$ Possibly, the modulation is thus an indication that at $V_{\mathrm{cg}} \approx 40 \mathrm{mV}$, the island splits up into two smaller, weakly coupled puddles, due to disorder. The modulated diamonds then reflect the formation of a "molecular state," or of a capacitance between the two puddles. Measurements on arrays of two puddles in parallel ${ }^{13}$ and of two as well as of three puddles in series ${ }^{14}$ have been reported. From those experiments, it is clear that more puddles than two would result in larger modulation periods. Since we cannot tune the two puddles independently nor the coupling between them, we are unable to extract the puddle sizes, the interdot conductance, or their configuration with respect to source and drain. Nevertheless, within this interpretation, the average CB period in Fig. 3(a) corresponds to $e / C_{g}$, where $e$ is the elemen- tary charge and $C_{g}$ the capacitance between the total island and the center gates. The average distance between the diamond corners along the $V_{b}$ direction corresponds to twice the charging energy $E_{C}=e^{2} / C_{\Sigma}$ of the island: $\delta V_{\text {sd }}=2 e / C_{\Sigma}$. Here, $C_{\Sigma}$ denotes the total capacitance of the island. From Fig. 3(a), we find $C_{\Sigma}=170 \mathrm{aF}$, which corresponds to a single electronic island radius of $r \approx 200 \mathrm{~nm}$. Hence, the level spacing inside the dot can be estimated as $\Delta \approx 8 \mu \mathrm{eV}$, which justifies our assumption of multilevel transport used earlier.

In summary, we have demonstrated that top gate defined nanostructures can be fabricated and operated on $p$-SiGe. The leakage currents can be significantly reduced by oxidizing the mesa edges before top gate deposition. We have observed Coulomb blockade on a top gate defined island in $p$ - $\mathrm{Si} / \mathrm{SiGe}$. The island is strongly disordered, which manifests itself in thermally enhanced transport as well as in a possible puddle formation at small hole densities.

The authors would like to thank S. Graf for technical assistance. Financial support by the Swiss National Science Foundation and MINAST is gratefully acknowledged.

\footnotetext{
${ }^{1}$ For a review, see L. P. Kouwenhoven, C. M. Marcus, P. L. McEuen, S. Tarucha, R. M. Westervelt, and N. S. Wingreen, in Proceedings of a NATO Advanced Study Institute, edited by L. P. Kouwenhoven, G. Schön, and L. L. Sohn (Kluwer, Dodrecht, Netherlands, 1997), Ser. E, Vol. 345, pp. 105-214.

${ }^{2}$ P. T. Coleridge, A. S. Sachiajda, H. Lafontaine, and Y. Feng, Phys. Rev. B 54, 14518 (1996).

${ }^{3}$ S. Madhavi, V. Venkataraman, J. C. Sturm, and Y. H. Xie, Phys. Rev. B 61, 15807 (2000).

${ }^{4}$ C. J. Emeleus, M. A. Sadeghzadeh, P. J. Phillips, EW. H. C. Parker, T. E. Whall, M. Pepper, and A. G. R. Evans, Appl. Phys. Lett. 70, 1870 (1997).

${ }^{5}$ M. A. Sadeghzadeh, Appl. Phys. Lett. 76, 348 (2000).

${ }^{6}$ P. W. Lukey, J. Caro, T. Zijlstra, E. van der Drift, and S. Radelaar, Phys. Rev. B 57, 7132 (1998); C. D. Akyüz, A. Zaslavsky, L. B. Freund, D. A. Syphers, and T. O. Sedgwick, Appl. Phys. Lett. 72, 1739 (1998).

${ }^{7}$ E. Leonbandung, L. Guo, Y. Wang, and S. Y. Chou, J. Vac. Sci. Technol. B 13, 2865 (1995).

${ }^{8}$ D. J. Paul, J. R. A. Cleaver, H. Ahmed, and T. E. Whall, Phys. Rev. B 49, 16514 (1994).

${ }^{9}$ V. Senz, T. Heinzel, T. Ihn, K. Ensslin, G. Dehlinger, D. Grützmacher, and U. Gennser, Phys. Rev. B 61, R5082 (2000).

${ }^{10}$ N. F. Mott, Proc. R. Soc. London, Ser. A 167, 348 (1949).

${ }^{11}$ A. L. Efros and B. I. Shklovskii, J. Phys. C 8, L49 (1975).

${ }^{12}$ C. H. Crouch, C. Livermore, R. M. Westervelt, K. L. Campman, and A. C. Gossard, Appl. Phys. Lett. 71, 817 (1997).

${ }^{13}$ F. Hofmann, T. Heinzel, D. A. Wharam, J. P. Kotthaus, G. Böhm, W. Klein, G. Tränkle, and G. Weimann, Phys. Rev. B 51, R13872 (1995).

${ }^{14}$ F. R. Waugh, M. J. Berry, D. J. Mar, R. M. Westervelt, K. L. Campman, and A. C. Gossard, Phys. Rev. Lett. 75, 705 (1995).
} 